\title{
Faraday rotation constraints on large scale Halo model
}

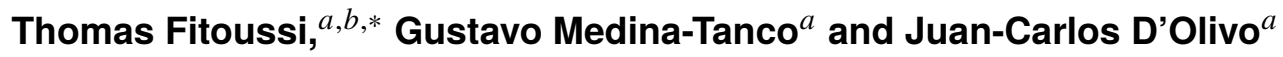 \\ ${ }^{a}$ Instituto de Ciencias Nucleares, UNAM, Circuito Exteriror S/N, Ciudad Universitaria, México D. F. \\ 0.4810, México \\ ${ }^{b}$ Karlsruhe Institute of Technology, Institut für Kernphysik, Karlsruhe, Germany \\ E-mail: thomas.fitoussi@kit.edu, gmtanco@correo.nucleares.unam.mx
}

The global structure of the magnetic field inside the disk of our Galaxy is reasonably well described by dynamo action and constrained by Faraday rotation measurements. The Halo, on the other hand, is much more of an enigma. Other face-on spiral galaxies show spiral magnetic structures in their disk, like the Milky Way, showing that our magnetic field is a fairly typical feature of this class of galaxies. Furthermore, RM-synthesis of CHANGE-ES observations shows an increasing number of edge-on spiral galaxies exhibiting $\mathrm{X}$-shaped structures surrounding the disk and extending orderly to distances of up to tens of kpc. Although the 3-dimensional topology of those magnetized halos and their physical nature is still unclear, they hint to the strong possibility that our galaxy also has a large and well organized magnetized Halo. The possible existence of an extended and topologically well organized magnetic field in the Halo has not been studied for the Milky Way. Specifically, the models in current use only take into account the Disk field, supplemented by a component that extends very little beyond the plane and decays rapidly with height. In this work, conceptually motivated by the possible existence of a Parker-type galactic outflow, we propose a simple Archimedean-like field, for an extended Halo magnetic field. We add this component to a simple disk magnetic field, in order to model the Faraday rotation signal of extragalactic sources as observed on Earth, for two different free electron density profiles, and compare the results with published maps of Faraday rotations measurements. We show that an extended magnetic field in the Halo is not only compatible with the observed Faraday rotations, but is actually favored by them.

$37^{\text {th }}$ International Cosmic Ray Conference (ICRC 2021)

July 12th - 23rd, 2021

Online - Berlin, Germany

\footnotetext{
${ }^{*}$ Presenter
} 


\section{Introduction}

Magnetic fields are ubiquitous in the Universe, from the scale of the solar systems to the full size of the Universe. The modeling of these magnetic fields is crucial for many astrophysical and cosmological (galaxy formation and evolution, transport of charged particles, ...). The Galactic magnetic field is a particular problem since it is linked to the large scale structure of the Galaxy and the interstellar medium (for a review see, for example, ref. [1]). Multiple observables can be used to constrain the Galactic magnetic field: starlight polarization, Faraday rotation from Galactic and extragalactic sources, Faraday tomography, diffuse synchrotron emission (radio and microwave) and diffuse dust emission. Each technique has its advantages and inconvenient and can probe one or two aspects of the magnetic field along the line of sight: strength (coherent or turbulent), amplitude parallel or perpendicular to the line of sight [2].

The modeling of the Galactic magnetic field includes two aspects: A coherent large-scale structure and small structures seen as turbulent on a larger scale [1,2]. The large-scale coherent magnetic field comprises the disk and halo fields. The disk component generally decreases exponentially with the height $z$ and inside the disk can be modeled with simple axisymmetric spirals [3]. This model allows to reproduce all the observables. In general, one adds to it or replace it by some spiral arms $[4,5]$. The structure of the magnetic field following or not the arms has been observed in other galaxies [6]. Moreover, a magnetic field structure with bissymetrical arms allows to reproduce the field reversal observed inside the disk. Observations of other galaxies with precise radio measurements made clear that a second $\mathrm{X}$-shape component is extending outside the disk inside the halo, whose origin is connected to the galactic dynamo and the galactic winds [2,6]. The fitting of the recent models including such kind of field tend to show that the Milky Way has indeed such a component [3-5]. A turbulent component can be added to reproduce the small scale structure that can impact on the large scale field. It also decomposes in two components: a purely isotropic field and an ordered (striated) magnetic field $[1,4]$.

Determining the magnetic field in the Milky Way is a difficult task. If the field structure in the disk is not clear, the situation is even worse for the halo. Most models use an ad hoc approach. Some issues, such as the extent and intensity of the halo field, play a crucial role in the propagation and identification of the source of high-energy cosmic rays. In a recent study [7], it has been indicated that other spiral galaxies have an extended magnetic field in the halo and beyond on distances of few kpc. To investigate how feasible it is that this is also the case for our galaxy, in this work we consider a simple model of the Galactic magnetic field, in which an extended halo field has been added to a simple disk field. We fit our model by comparing the predicted Faraday rotation for two free electron densities with the measurements for extra galactic sources. As we show, the extended halo field is favored by observations.

\section{Galactic magnetic field modeling}

We model the Galactic magnetic field $\mathbf{B}$ as a linear superposition of the disk field $\mathbf{B}_{\mathrm{D}}$ and the halo field $\mathbf{B}_{\mathrm{H}}$ :

$$
\mathbf{B}=\mathbf{B}_{\mathrm{D}}\left(1-T\left(z, z_{0}\right)\right)+\mathbf{B}_{\mathrm{H}} T\left(z, z_{0}\right),
$$


where $T\left(z, z_{0}\right)=1 /\left(1+\exp \left(-k\left(|z|-z_{0}\right)\right)\right)$ is a smooth step function represented by the logistic function. where $z_{0}$ is the altitude of the halo and $k$ is the logistical function factor (fixed at $50 \mathrm{kpc}^{-1}$. In this way, we guarantee a continuous transition between the disk and the halo, like in Jansson and Farrar [4].

$\mathbf{B}_{\mathrm{D}}$ and $\mathbf{B}_{\mathrm{H}}$ have both a regular and a turbulent component. Since the observed Faraday rotations are due to the regular components, although the turbulent is larger, in what follows we will not consider it. The disk and the halo field are parametrized independently. We use a cylindrical $(r, \varphi, z)$ coordinate system for the disk and a spherical $(\rho, \theta, \varphi)$ coordinate system for the halo, both with the origin at the Galactic center.

The disk field is parallel to the $x y$ plane and has a shape similar to the spiral structure of the Galaxy. Here, we consider the bisymmetric version of the logarithmic spiral model, with opposite direction of the field in different arms, as in Pshirkov et al. [8]:

$$
\mathbf{B}_{\mathrm{D}}=\left(\sin p \hat{\boldsymbol{u}}_{\boldsymbol{r}}+\cos p \hat{\boldsymbol{u}}_{\varphi}\right)\left[\frac{B_{0}^{\mathrm{D}}}{\cos \Phi} \cos \left(\varphi-b \ln \frac{r}{r_{\odot}}+\Phi\right)\right] \times \begin{cases}r_{\odot} / r_{c}, & \text { if } r<r_{c} \\ r_{\odot} / r, & \text { if } r \geq r_{c}\end{cases}
$$

where $B_{\mathrm{D}}^{0}$ is the local field strength at the Sun position, $r_{\odot}$ is the Galactocentric distance of the Sun, taken as $8.5 \mathrm{kpc}, b=1 / \tan p$ and $\Phi=b \ln \left(1+d / r_{\odot}\right)-\frac{\pi}{2}$. Here, $d$ is the distance for the first field reversal, $p$ is the pitch angle and $r_{c}$ the characteristic radius of the central region where the field is assumed constant $\left(r_{c} \geq 1 \mathrm{kpc}\right)$.

Regarding the halo field, we assume that it consists of an X-shape field and a toroidal field [4, 5] and modeled it with an Archimedean spiral [9] that is consistent with a large scale magnetic field due to winds:

$$
\mathbf{B}_{\mathrm{H}}= \begin{cases}0, & \rho<\rho_{0} \\ \frac{|z|}{z} B_{0}^{\mathrm{H}}\left(\frac{\rho_{0}}{\rho}\right)^{2}\left(\hat{\boldsymbol{u}}_{\boldsymbol{r}}-\frac{\rho}{\rho_{1}} \sin \theta \hat{\boldsymbol{u}}_{\varphi}\right), & \rho \geq \rho_{0}\end{cases}
$$

where $B_{0}^{\mathrm{H}}$ is the magnetic field strength, $\rho_{0}$ is the spherical radius at which $B_{0}^{\mathrm{H}}$ is defined, and $\rho_{1}$ is the typical distance where the halo goes from a radial to an azimuthal field. The factor $|z| / z$ accounts for the reversal of the field directions above and below the Galactic plane, which reproduces the dipole structure observed with Faraday measurements.The lines leave the origin in the north hemisphere and re-enter from below in the south hemisphere (see figure 2). The field rotates clockwise instead of anti-clockwise above the disk (this is equivalent to take $\omega$ negative in Parker [9]).

\section{Fitting results}

There are many ways to fit a Galactic magnetic field model. In this article, we will use only Faraday rotation measurements from extragalactic sources. We will use the full sky Faraday rotation map published by Oppermann et al. [10] which contains 196608 pixels (resolution of $0.45^{\circ}$ ). For the purpose of our fit we reduce the resolution to $7.32^{\circ}$ (768 pixels). In each pixel is taken the median of 256 pixels from the original map included inside the given pixel. The uncertainty $\sigma_{i}$ for each pixel is computed as $\sigma_{i}^{2}=\sqrt{\sum_{j} \sigma_{j}^{2}} / N_{i}$, where $\sigma_{j}$ is the uncertainty in each sub-pixel and $N_{i}$ the number of sub-pixels (256). 
To compute the Faraday rotation measurements, we need a model for the free electron density. We used the model NE2001 of Cordes and Lazio [11], which integrates four spiral arms, a thick and a thin disk, the Galactic center, the local interstellar medium and some specific clumps and voids. We use the thick disk height at $1.31 \mathrm{kpc}$ (instead of $0.95 \mathrm{kpc}$ ) and the midplane density at $0.016 \mathrm{~cm}^{-3}$ (instead of $0.035 \mathrm{~cm}^{-3}$ ) according to corrections for pulsars observations [12]. We also consider the new model from Yao et al. [13] (here after YMW17).

We aim to examine the limits of the halo in term of amplitude and structure. Our halo model only depends on three parameters (eq. 3): the normalized amplitude $B_{0}^{\mathrm{H}}$, a normalisation distance $\rho_{0}$ and $\rho_{1}$ that can be seen as the typical distance from the Galactic center where the field changes from a radial to a toroidal field. To study the halo field we must also take into account the effect of $\mathbf{B}_{\mathrm{D}}$. To reduce the computation time required to set the disk field and instead use it to test as many parameters as possible for the halo, we set some parameters for $\mathbf{B}_{\mathrm{D}}$ and test a few number of values for the rest of them according to the known results. The amplitude of the disk magnetic field at the Earth is set at $B_{0}^{\mathrm{D}}=1.5 \mu \mathrm{G}$ [14]. The best value from Pshirkov et al. [8] $R_{c}=5 \mathrm{kpc}$ is used. We only tested three values for $d(1,0$ and $-1 \mathrm{kpc})$ to have a crude idea if the distance of the first field reversal is more in the direction of the Galactic center or to the outside of the Galaxy from Earth. The pitch angle at Earth is not clearly defined depending on the technique used. Then for the fitting, we let $p$ equal to $-6,-8$ and $-10^{\circ}$. Finally, the thickness of the disk $z_{0}$ is not expected to be extremely large $[4,8]$. For safety we let it take the values $0.5,1$ and $1.5 \mathrm{kpc}$.

Concerning the halo, we tested the amplitude $B_{0}^{\mathrm{H}}$ between 0.1 and $60 \mu \mathrm{G}$ with a logarithmic step of 0.1 . The parameter $\rho_{0}$ corresponds both to the size of the sphere outside of which the magnetic field is defined and the normalization distance of $B_{0}^{\mathrm{H}}$, it was tested for values between 1 and $5 \mathrm{kpc}$ (steps of $1 \mathrm{kpc}$ ) corresponding to the size of the Galactic bulk. $\rho_{1}$ was tested as a multiple of $\rho_{0}$ between 1 and 20 times $\rho_{0}$, with a logarithmic step of 0.1 .

For each set of parameters, we evaluate the quality of the modeling compared to the map of Faraday rotation measurements using the $\chi^{2}$ method. We also use the reduced $\chi^{2}: \chi_{\text {red }}^{2}=\chi^{2} /\left(N_{\text {pix }}-N_{d o f}\right)$, where $N_{\text {pix }}$ is the number of pixels from the Faraday rotation map and $N_{d o f}$ is the number of degree of freedom in the model ( 5 for the disk and 3 for the halo). If $\chi_{\text {red }}^{2}$ could be high, we should see that the uncertainties from Oppermann et al. [10] are high. Then, it is more an indication of an improvement of the fitting rather than an indication of a goodness of fitting.

In table 1 we summarized the values tested for each parameter and the best fit obtained for the two free electron density model NE2001 [11] and YMW17 [13] (between paranthesis are the values that can be acceptable). Since we tested only few values for the disk, it is hard to draw strong conclusions. Also, since our model of the disk is sligthly different (attenuation in $z$ is a logistical function instead of an exponential), it is hard to compare with Pshirkov et al. [8], but for the pitch angle that is used the same way we get the same estimate of $p=-6^{\circ}$. The best fit for $d$ is $-1 \mathrm{kpc}$ going also in the same direction as Pshirkov et al. [8] (-0.6 kpc). Concernig $z_{0}$, we found a best fit of -0.5 instead of $-1 \mathrm{kpc}$ but since the cut-off is different (logistical function against exponential), they are hard to say if it is comparable.

The parameters of the halo are less constrained. In particular, the best fit for the amplitude $B_{0}^{\mathrm{H}}$ is 


\begin{tabular}{llrrr}
\hline Parameter & Units & Values tested & \multicolumn{2}{c}{ best fits } \\
& & & NE2001 [11] & YMW17 [13] \\
\hline \hline$\chi^{2}\left(\chi_{\text {red }}^{2}\right)$ & & & $2186(2.88)$ & $3445(4.53)$ \\
\hline disk & & & & \\
$B_{0}^{\mathrm{D}}$ & {$[\mu \mathrm{G}]$} & 1.5 & 1.5 & 1.5 \\
$R_{c}$ & {$[\mathrm{kpc}]$} & 5 & 5 & 5 \\
$\mathrm{~d}$ & {$[\mathrm{kpc}]$} & $-1,0,1$ & -1 & -1 \\
$\mathrm{p}$ & {$[\mathrm{deg}]$.} & $-6,-8,-10$ & $-6(-8)$ & -6 \\
$z_{0}$ & {$[\mathrm{kpc}]$} & $0.5,1,1.5$ & 0.5 & $0.5(1)$ \\
\hline halo & \multicolumn{4}{c}{} \\
$B_{0}^{\mathrm{H}}$ & {$[\mu \mathrm{G}]$} & {$[0.1,60](\log$ step 0.1) } & $10(8 \rightarrow 15)$ & $10(7 \rightarrow 15)$ \\
$\rho_{0}$ & {$[\mathrm{kpc}]$} & {$[1,5](\mathrm{step} 1)$} & $1(2)$ & $1(2)$ \\
$\rho_{1}$ & {$\left[\times \rho_{0}\right]$} & {$[1,20](\log$ step 0.1) } & $1(1 \rightarrow 5)$ & $1(1 \rightarrow 5)$ \\
\hline
\end{tabular}

Table 1: Table of the best fit parameters (see text for details)

between 1 and $15 \mu \mathrm{G}, \rho_{0}$ tend to favour small values ( 1 or $2 \mathrm{kpc}$ ). Interestingly for each best value of $\rho_{0}$ we find a good fit for $\rho_{1}<5 \rho_{0}$ indicating the necessity of an X-field near to the Galactic center $\left(\rho_{0}<\rho<\rho_{1}\right)$ and then a magnetic field spining at larger distance.

In fig. 1 (center and right) are represented the Faraday rotation maps corresponding to the best fit seen from Earth for both free electron densities, NE2001 [11] and YMW17 [13]. The Faraday rotation map produced by the best fit reproduces the global structure observed on the map of Oppermann et al. [10] (fig. 1 left): A quadrupole inside the disk $\left(|b|<10^{\circ}\right)$ and a dipole in the southern and northern hemispheres. The dipole in the south is more extended than in the north. We obtain the same kind of results for both electron densities, but the $\chi^{2}$ is much better in the case of NE2001 [11]. In fact, the best fit map for YMW17 [13] shows a higher mean value of the Faraday rotation, which could be associated with the higher density of electrons in the disk. Moreover, some features, like the red spot near the disk at $l \simeq-75^{\circ}$, are less visible for the same reason.
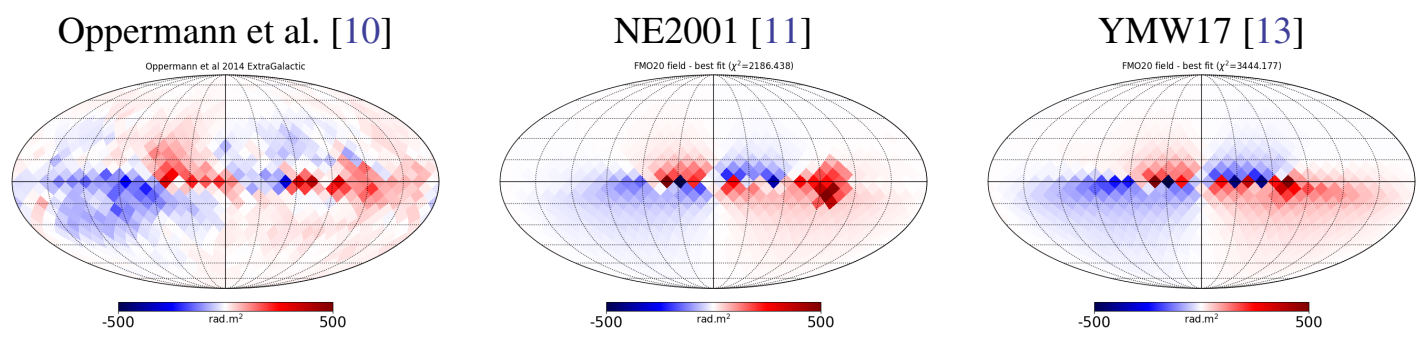

Figure 1: Map of the Faraday rotation from Oppermann et al. [10] (left) and for the best fit with the NE2001 [11] model (center) and the YMW17 [13] model (right) of the free electron density.

In figure 2 are represented two maps of the best fit magnetic field (planes $\mathrm{z}=0$ and $\mathrm{y}=0$ ). The $\mathrm{X}$-field is clearly visible as well as the extended structure observed in other spiral Galaxies [7]. 

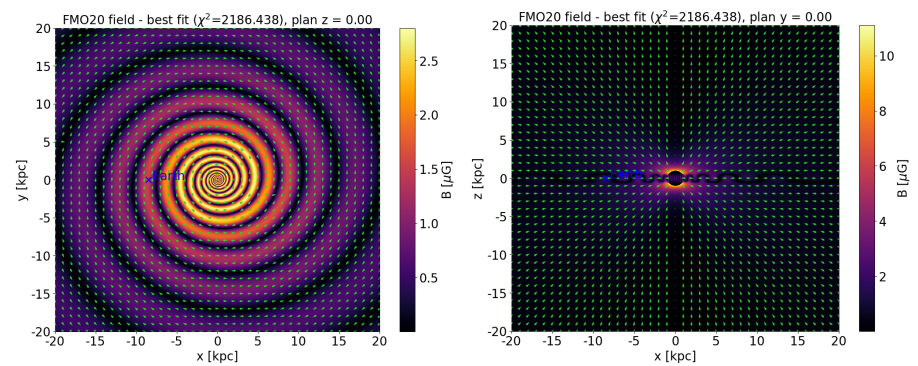

Figure 2: Map ( $\mathrm{z}=0$ and $\mathrm{y}=0$ ) of the magnetic field for the best fit.

\section{Limits on the halo}

As indicated in the previous section, we have only tested some values of the disk's field parameters. As a result, a single set of such parameters emerged as the "best fit". Here, we will take that set as the best model for the actual field in the disk and determine to what extent it is possible to improve the fit by adding the halo field modeled above. To do this, we will examine whether with this inclusion it is possible to get a total magnetic field whose $\chi^{2}$ is smaller compared to the $\chi_{\text {disk }}^{2}$ obtained by adjusting only the field of the disk.

In figure 3 we show the $\chi^{2}$ (color map) as a function of the halo parameters, $\rho_{0}, \rho_{1}$, and $B_{0}^{\mathrm{H}}$. Each of the figures is made for a value of $\rho_{0}(1,2,3 \mathrm{kpc})$ and for a given free electron density model (NE2001 on top and YMW17 on bottom). Each colored area represents the parameter space where the $\chi^{2}$ of the full model (disk + halo) is a certain percentage $(100 \%, 95 \%, 90 \%, 85 \%, 82 \%, 80 \%$, $78 \%$ ) below the best fit of the disk only model in the full sky. The blue and purple areas represent the parameter space where adding our halo model improves the fit significantly, while the green area shows the parameters for which there is a little improvement. The yellow, orange, and red areas represent successive degradation of the fit where the full models are more or less equivalent to the disk only model.

The blue area for a better fit shows some degeneracy, with a maximum value of the magnetic field of a few tens of microgauss and a distance $\rho_{1}$ of few kpc. We can see that in the purple area (where the improvement of the model with a halo is noticeable), there is a clear dependence between $B_{0}^{\mathrm{H}}$, $\rho_{0}$ and $\rho_{1}$. From the dashed and dotted lines, the dependency can be expressed approximately by the following equation:

$$
B_{H}^{0}(\text { best })=7\left(\frac{\rho_{0}}{1 k p c}\right)^{-1}\left(\frac{\rho_{1}}{\rho_{0}}\right) \mu G
$$

Indeed increasing the amplitude of the magnetic field requires to increase the distance $\rho_{1}$ or decrease $\rho_{0}$. This means that for a stronger magnetic field, the field will start to become toroidal at a larger distance from the Galactic center. A large value of the magnetic field might seem inadequate, but it should be noted that it is confined near the Galactic center and decreases rapidly as $1 / \rho^{2}$ (in spherical coordinates) on small scales. At greater distance, the field amplitude varies as $1 / \rho$ and, therefore, our model suggests that at tens of kpc from the galactic plane, a magnetic field of a few microgauss could still exist in the halo. A large amplitude is not completely impossible because at such large distance the free electron density is too low to probe the magnetic field. 

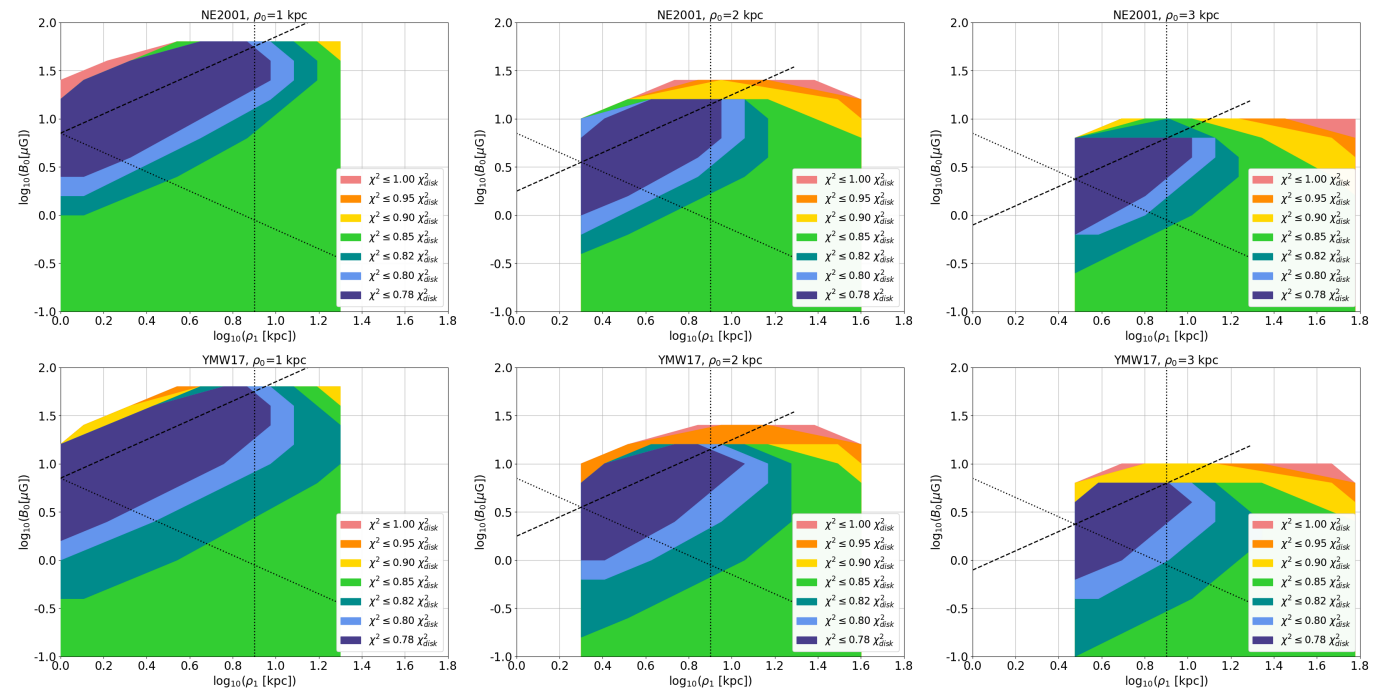

Figure 3: Parameters space of the halo field versus the reduced $\chi^{2}$. Colored areas give the fluctuation of the $\chi^{2}$ compare to the best fit with only the disk field.

\section{Conclusions}

In this work, we propose an Archimedean field for an extended halo magnetic field. Such a field added to a simple magnetic field of the disk was used to calculate the Faraday rotation of extragalactic sources. The good agreement of the values predicted by our model with published Faraday rotation maps suggests that a magnetic field of a few microgauss could exist in the halo at a large distance from the galactic plane. Such a field could not be probed at great distance through Faraday rotations due to the absence of free electrons. If such a field exists, it would have significant implications in extragalactic astrophysics. In particular, the deflection of ultra-high energy cosmic rays within the halo would be more important than expected and would have a significant impact on the determination of sources.

\section{References}

[1] Marijke Haverkorn. Magnetic Fields in the Milky Way. arXiv:1406.0283 [astro-ph], 407: 483-506, 2015. doi: 10.1007/978-3-662-44625-6_17. URL http://arxiv.org/abs/ 1406.0283. arXiv: 1406.0283.

[2] Tess R. Jaffe. Practical Modeling of Large-Scale Galactic Magnetic Fields: Status and Prospects. Galaxies, 7(2):52, June 2019. doi: 10.3390/galaxies7020052. URL https: //wwW . mdpi . com/2075-4434/7/2/52.

[3] X.-H. Sun and W. Reich. The Galactic halo magnetic field revisited. Research in Astronomy and Astrophysics, 10:1287-1297, December 2010. ISSN 1674-4527. doi: 10.1088/1674-4527/ 10/12/009. URL http: //adsabs . harvard. edu/abs/2010RAA . . . 10.1287S. 
[4] R. Jansson and G. R. Farrar. A New Model of the Galactic Magnetic Field. The Astrophysical Journal, 757(1):14, 2012. ISSN 0004-637X. doi: 10.1088/0004-637X/757/1/14. URL http: //stacks.iop.org/0004-637X/757/i=1/a=14.

[5] P. Terral and K. Ferrière. Constraints from Faraday rotation on the magnetic field structure in the Galactic halo. Astronomy and Astrophysics, 600:A29, 2017. doi: 10.1051/0004-6361/ 201629572. URL https://ui . adsabs.harvard.edu/abs/2017A\%26A . . 600A . .29T/ abstract.

[6] R. Beck and R. Wielebinski. Magnetic Fields in Galaxies. Planets, Stars and Stellar Systems. Volume 5: Galactic Structure and Stellar Populations, page 641, 2013. doi: 10.1007/978-94-007-5612-0_13. URL http://adsabs.harvard.edu/abs/2013pss5. book. . 641B.

[7] M. Krause et al. CHANG-ES XXII: Coherent Magnetic Fields in the Halos of Spiral Galaxies. arXiv e-prints, 2004:arXiv:2004.14383, April 2020. URL http://adsabs . harvard.edu/ abs/2020arXiv200414383K.

[8] M. S. Pshirkov, P. G. Tinyakov, P. P. Kronberg, and K. J. Newton-McGee. Deriving the Global Structure of the Galactic Magnetic Field from Faraday Rotation Measures of Extragalactic Sources. The Astrophysical Journal, 738(2):192, 2011. ISSN 0004-637X. doi: 10.1088/ 0004-637X/738/2/192. URL http://stacks. iop.org/0004-637X/738/i=2/a=192.

[9] E. N. Parker. Dynamics of the Interplanetary Gas and Magnetic Fields. The Astrophysical Journal, 128:664, November 1958. ISSN 0004-637X, 1538-4357. doi: 10.1086/146579. URL http: //adsabs . harvard. edu/doi/10.1086/146579.

[10] N. Oppermann et al. Estimating extragalactic Faraday rotation. Astronomy \& Astrophysics, 575:A118, 2015. ISSN 0004-6361, 1432-0746. doi: 10.1051/0004-6361/201423995. URL http: //www . aanda.org/10.1051/0004-6361/201423995.

[11] J. M. Cordes and T. J. W. Lazio. NE2001.I. A New Model for the Galactic Distribution of Free Electrons and its Fluctuations. 2002. URL https://arxiv.org/abs/astro-ph/ $0207156 \mathrm{v} 3$.

[12] Modelling the Galactic distribution of free electrons: The Galactic distribution of free electrons. 427. ISSN 00358711, 13652966.

[13] J. M. Yao et al. A NEW ELECTRON-DENSITY MODEL FOR ESTIMATION OF PULSAR AND FRB DISTANCES. The Astrophysical Journal, 835, January 2017. ISSN 0004-637X. doi: 10.3847/1538-4357/835/1/29. URL https://doi.org/10.3847\%2F1538-4357\% 2F $835 \% 2$ F $1 \% 2$ F 29 .

[14] J. L. Han et al. Pulsar rotation measures and the large-scale structure of Galactic magnetic field. The Astrophysical Journal, 642:868-881, May 2006. ISSN 0004-637X, 1538-4357. doi: 10.1086/501444. URL http://arxiv.org/abs/astro-ph/0601357. arXiv: astro$\mathrm{ph} / 0601357$. 